\title{
Spontaneous Electron Emission from Hot Silver Dimer Anions: Breakdown of the Born-Oppenheimer Approximation
}

\author{
E. K. Anderson, ${ }^{1, *}$ A. F. Schmidt-May $\odot,{ }^{2,1}$ P. K. Najeeb $\odot,{ }^{1}$ G. Eklund, ${ }^{1}$ K. C. Chartkunchand $\odot,{ }^{3}$ \\ S. Rosén, ${ }^{1} \AA$ A. Larson, ${ }^{1}$ K. Hansen $\odot,{ }^{4,5}$ H. Cederquist $\odot,{ }^{1}$ H. Zettergren $\odot,{ }^{1}$ and H. T. Schmidt $\oplus^{1, \dagger}$ \\ ${ }^{1}$ Department of Physics, Stockholm University, AlbaNova, SE-106 91 Stockholm, Sweden \\ ${ }^{2}$ Institut für Ionenphysik und Angewandte Physik, Universität Innsbruck, A-6020 Innsbruck, Austria \\ ${ }^{3}$ Atomic, Optical, and Molecular Physics Laboratory, RIKEN Cluster for Pioneering Research Wako-shi, Saitama 351-0198, Japan \\ ${ }^{4}$ Center for Joint Quantum Studies and Department of Physics, Tianjin University, 92 Weijin Road, Tianjin 300072, China \\ ${ }^{5}$ Department of Physics, University of Gothenburg, 41296 Gothenburg, Sweden
}

(Received 20 November 2019; revised manuscript received 15 March 2020; accepted 9 April 2020; published 27 April 2020)

\begin{abstract}
We report the first experimental evidence of spontaneous electron emission from a homonuclear dimer anion through direct measurements of $\mathrm{Ag}_{2}^{-} \rightarrow \mathrm{Ag}_{2}+e^{-}$decays on milliseconds and seconds timescales. This observation is very surprising as there is no avoided crossing between adiabatic energy curves to mediate such a process. The process is weak, yet dominates the decay signal after $100 \mathrm{~ms}$ when ensembles of internally hot $\mathrm{Ag}_{2}^{-}$ions are stored in the cryogenic ion-beam storage ring, DESIREE, for $10 \mathrm{~s}$. The electron emission process is associated with an instantaneous, very large reduction of the vibrational energy of the dimer system. This represents a dramatic deviation from a Born-Oppenheimer description of dimer dynamics.
\end{abstract}

DOI: 10.1103/PhysRevLett.124.173001

In the Born-Oppenheimer approximation for molecules, the electronic and nuclear degrees of freedom are treated separately [1]. This is the basis for representing molecular quantum states as products of electronic and vibrationalrotational wave functions. Strictly within such a description, energy bound in vibrational excitation cannot be transferred to the electronic degrees of freedom. For complex systems, rapid transfers of energy between electronic and nuclear degrees of freedom are nevertheless common. The energy transfer may happen when such systems are photoexcited and evolve from an electronically excited potential-energy surface via one or more conical intersections to the ground state [2]. The reverse process-inverse internal conversion-where a vibrationally hot complex system in the electronic ground state converts vibrational energy to electronic excitations is possible as well and may lead to recurrent fluorescence processes [3-6]. Rapid transfer of energy between the electronic and nuclear degrees of freedom is also expected for most small metal cluster anions [7-9]. Typically, such processes occur on picosecond timescales at avoided crossings or conical intersections between adiabatic potential-energy curves or surfaces. Recently, electron emission from excited sulfur hexafluoride anions was ascribed to a crossing of the anion and neutral diabatic

Published by the American Physical Society under the terms of the Creative Commons Attribution 4.0 International license. Further distribution of this work must maintain attribution to the author(s) and the published article's title, journal citation, and DOI. Funded by Bibsam. potential-energy surfaces [10], yielding a strong coupling to the electronic continuum states.

For diatomic systems with no avoided crossings between adiabatic potential-energy curves to drive the transition, the situation is fundamentally different [11]. Still, for $\mathrm{Ag}_{2}^{-}$an electron emission process must be accompanied by a dramatic decrease in vibrational excitation energy by an amount exceeding the $\mathrm{Ag}_{2}$ electron affinity, $\mathrm{EA}=1.023 \pm 0.007 \mathrm{eV}$ [12]. The weakness of such a coupling between nuclear and electronic degrees of freedom for another diatomic system, the cyano radical $(\mathrm{CN})$, was explicitly discussed by Douguet et al. [13]. They pointed out that indirect radiative electron attachment, where the binding energy of the captured electron would be balanced by a vibrational excitation, was insignificant precisely because such a process would involve a very large deviation from a Born-Oppenheimer description. Molecular formation in ultracold gases via Feshbach resonances is a conceptually related process, which also involves a significant transfer of energy between nuclear and electronic degrees of freedom in the absence of a curve crossing [14].

In this Letter, we report the first direct observation of electron emission from a homonuclear dimer anion. We measure the spontaneous unimolecular decays of internally hot $\mathrm{Ag}_{2}^{-}$anions stored in the cryogenic Double Electrostatic Ion-Ring Experiment (DESIREE) $[15,16]$. In the experiment, we separate the contributions from fragmentation $\mathrm{Ag}_{2}^{-} \rightarrow$ $\mathrm{Ag}+\mathrm{Ag}^{-}$and electron emission $\mathrm{Ag}_{2}^{-} \rightarrow \mathrm{Ag}_{2}+e^{-}$. We find that the former process dominates the decay signal up to $100 \mathrm{~ms}$ of storage, while the latter process, very surprisingly, dominates the signal thereafter. 


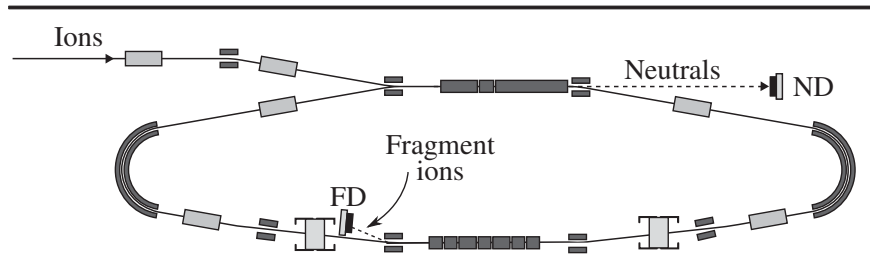

FIG. 1. Schematic of the electrostatic ion-beam storage ring used for the present measurements [15,16]. A $10 \mathrm{keV} \mathrm{Ag}_{2}^{-}$silver dimer anion bunch of about $10^{5}$ ions is injected to circulate in a closed orbit at a revolution frequency of $10.8 \mathrm{kHz}$. Neutral particles $\mathrm{Ag}$ and $\mathrm{Ag}_{2}$ from spontaneous fragmentation and from electron-detachment processes in the straight section closest to the injection port are detected by the ND. Atomic anions, $\mathrm{Ag}^{-}$, from fragmentation events in the opposite straight section are detected by the FD.

In Fig. 1, we show a schematic of one of the DESIREE $[15,16]$ ion-beam storage rings with its detectors for neutrals [neutral detector (ND)] and for fragments [fragment detector (FD)]. The ring is operated at cryogenic temperatures and with a residual-gas density of only a few $\mathrm{H}_{2}$ molecules per $\mathrm{mm}^{3}$. This enables ion storage for tens of minutes [17]. A cesium sputter ion source with a silver cathode is used to produce silver cluster anions in high vibrational and rotational states. These anions are accelerated to $10 \mathrm{keV}$. A $90^{\circ}$ analyzing magnet is then used to select a $150-200 \mathrm{pA}{ }^{107} \mathrm{Ag}_{2}^{-}$beam for injection into the ring, yielding about $10^{5}$ stored ions. This low current is used in order to avoid saturation of the detectors.

Fragmentation and electron detachment are the only two pathways for the rotationally and vibrationally excited dimer anions to spontaneously decay. Rovibrationally excited ${ }^{107} \mathrm{Ag}_{2}^{-}$ions in the electronic ground state cannot relax radiatively through electric-dipole transitions. Higher multipole order decays are possible, but are expected to be extremely slow-no calculation for $\mathrm{Ag}_{2}^{-}$exists, but for another homonuclear dimer $\mathrm{H}_{2}^{+}$the corresponding lifetimes have been calculated to be of the order of days [18]. Consistent with these expectations, no radiative cooling is observed for the silver dimer anions in the present experiment.

The rates of neutral products $\left(\mathrm{Ag}_{2}, \mathrm{Ag}\right)$ from fragmentation and electron detachment in one of the straight sections of the ring (Fig. 1) are measured as functions of time after ion production using the ND detector. The FD detector is used to measure the rate of $\mathrm{Ag}^{-}$products, which is due to fragmentation events only (Fig. 1). All data are recorded in list mode with the time after ion formation in the source recorded for each detection event.

The measured count rates of neutrals (Ag from fragmentation and $\mathrm{Ag}_{2}$ from electron emission) and fragment ions $\left(\mathrm{Ag}^{-}\right.$from fragmentation) leaving the ensembles of stored $\mathrm{Ag}_{2}^{-}$ions are shown in the upper panel of Fig. 2. The FD and ND detectors are equivalent: both are $40 \mathrm{~mm}$ diameter microchannel-plate detector assemblies with

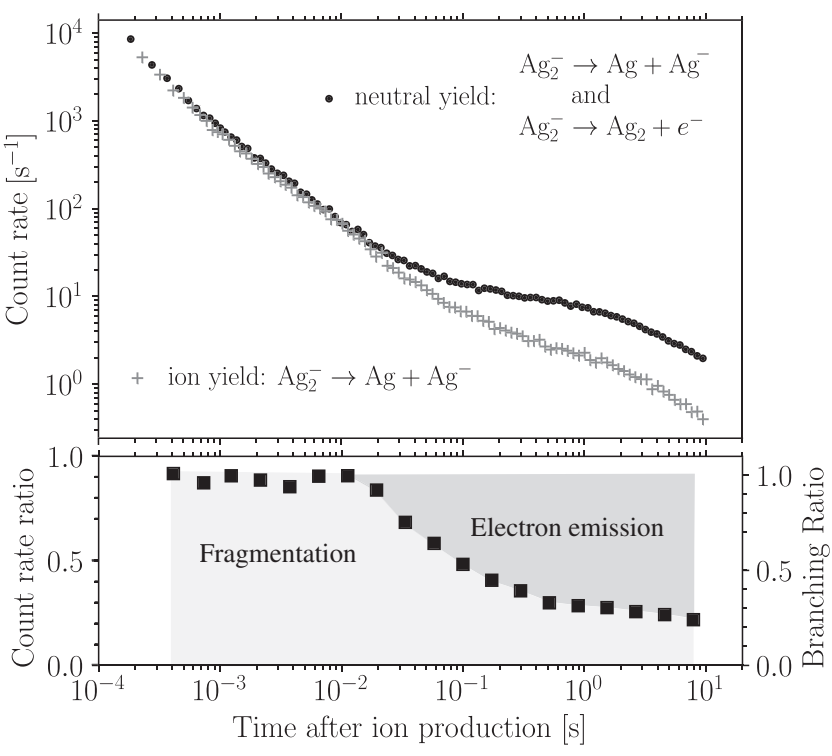

FIG. 2. Data recorded with $\approx 10^{5}$ stored $10 \mathrm{keV} \mathrm{Ag}_{2}^{-}$anions. (Upper) Background corrected detector count rates as functions of time after the ion production in the Cs sputter ion source: •, neutral products recorded by the neutral detector (ND in Fig. 1); + , ionic $\mathrm{Ag}^{-}$fragments recorded by the fragment detector (FD in Fig. 1). (Lower) Ratio between the $\mathrm{Ag}^{-}$and $\left(\mathrm{Ag}+\mathrm{Ag}_{2}\right)$ count rates as measured by the FD and ND detectors, respectively (left vertical axis). The vertical axis to the right has been corrected for the slightly different detection efficiencies of the two detectors (see text) and thus shows the branching ratio for fragmentation directly. The statistical errors are smaller than the symbols in both panels.

resistive anodes. For this reason, the two count rates are directly displayed without any normalization in the upper panel of Fig. 2. The zero point for the timescale is set to be the moment of formation of the ions in the cesium sputter ion source, and the data are binned with bin widths increasing linearly with time for clear presentation on a logarithmic scale, with the exception of the first few data points, which each represent count rates for single revolutions of $93 \mu$ s duration. The detector dark count background rates are measured prior to ion-beam injection to be $6.27 \pm 0.03$ and $4.32 \pm 0.03 \mathrm{~s}^{-1}$ for the neutral and fragment detectors, respectively, and have been subtracted from the data shown in Fig. 2. Up to $10 \mathrm{~ms}$ after the ensemble of stored ${ }^{107} \mathrm{Ag}_{2}^{-}$ions were formed, we see that the two detectors have very similar count rates and identical time dependences. We take this as evidence that the fragmentation process is completely dominating yielding equal numbers of neutral-atom and atomic-anion products within this time range.

In the lower panel of Fig. 2, we show the ratio of the count rates on the fragment detector and the neutral detector and we notice that this ratio has a constant value of $0.88 \pm 0.02$ for $t<10 \mathrm{~ms}$. The deviation from unity can be ascribed to a small difference in the detection efficiencies of the two detectors. For $t>10 \mathrm{~ms}$, the two detectors' count 
rates deviate strongly, with the neutral detector rate being about 5 times that of the fragment detector at $t=10 \mathrm{~s}$. The right-hand vertical axis of the lower panel of Fig. 2 is corrected for the slightly different detection efficiencies of the two detectors and thus directly gives the fraction of the decays that are due to fragmentation as a function of time. It is clearly demonstrated that, while fragmentation dominates initially, the contribution from electron detachment falls off more slowly with time and therefore becomes visible at around $t=10 \mathrm{~ms}$ and, in fact, dominates for $t>100 \mathrm{~ms}$ in the present experiment.

When interpreting decay curves like the ones in Fig. 2, it is important to emphasize that the large reduction in count rate by many orders of magnitude over the first few tens of milliseconds does not mean that almost all stored ions have decayed. In fact, a comparison of the numbers of injected ions and of detected fragments (taking into account geometrical limitations and detection efficiencies) indicate that about $99.9 \%$ of the injected ions have not decayed after $10 \mathrm{~s}$ in the present experiment. Those ions, which are either stable or decay with very low decay rates due to lower internal energies, can, however, still contribute to the neutral-particle signal by residual-gas collisions. The 3-4 orders of magnitude reduction of the residual-gas density compared to room-temperature storage rings [15] is therefore absolutely crucial for studies of the $\mathrm{Ag}_{2}^{-}$decays for seconds-long storage times.

In pioneering work on silver dimer anion decays in the room-temperature Electrostatic Ion-Storage Ring, Aarhus (ELISA) [19], the neutral-particle signal could only be followed for $80 \mathrm{~ms}$ due to the significant background from neutral products of collisions between the stored ion beam and the residual gas [20]. Our observation, that the count rate of neutrals is dominated by fragmentation early during storage, confirms the interpretation given in Ref. [20], namely, that their signal is due to fragmentation of dimer anions. This also strongly supports their conclusion [20] that ions with very high angular momentum and with sufficient vibrational excitation for tunneling through the centrifugal barrier are responsible for the fragmentation signal.

In Fig. 3, we show, as examples, approximate electronic ground state potential-energy curves for the neutral silver dimer and for the dimer anion for nonrotating $L=0$ systems (left panel) and for dimers with a high angular momentum $L=300$ (right panel). Rates for tunneling through the centrifugal barrier, schematically indicated in the right panel of Fig 3, were calculated in [20]. These rates were found to depend very strongly on the vibrational and rotational quantum numbers of the large number of rovibrational levels, thereby leading to a quasicontinuum of decay rates and thus the power-law decays observed both here and in earlier experiments [20,21].

The potential-energy curves shown in Fig. 3 are Morse potentials generated with parameter values from [12,22]. Dimer anions occupying levels with modest angular
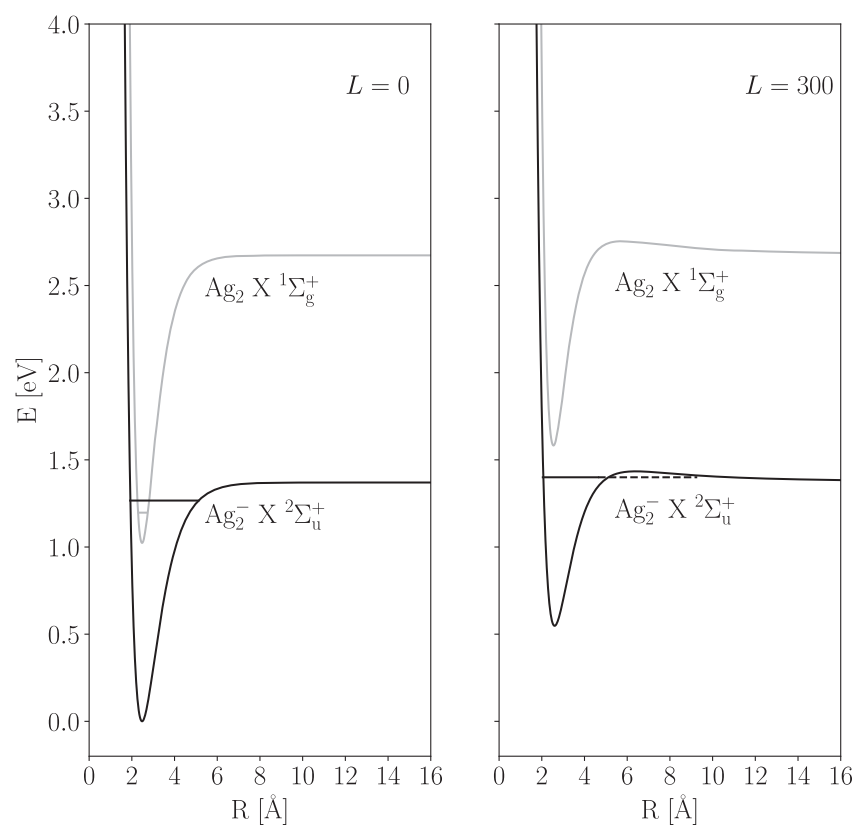

FIG. 3. Approximate potential-energy curves based on literature values from [12,22] for rotational angular momentum $L=0$ (left) and $L=300$ (right). Black curves, $L=0$ and $L=300$ dimer anion electronic ground state potentials; gray curves, equivalent electronic ground state potentials of the neutral dimer. The observed fragmentation is interpreted as due to tunneling through the centrifugal barrier for dimer anions in high angular-momentum levels as suggested in Ref. [20]. The observed electron-detachment signal is ascribed to dimer anions in high vibrational levels with too low angular momenta to fragment but sufficient vibrational energy to detach the electron by the weak coupling of vibrational and electronic degrees of freedom (see text). The vibrational levels indicated in the left panel are $v=110$ for the anion and $v=7$ for the neutral dimer. The metastable rovibrational level illustrating the tunneling fragmentation as an example in the right panel has $L=300$ and $v=88$.

momenta can accommodate high vibrational excitations and yet be energetically prohibited from fragmenting. As the electron affinity of $\mathrm{Ag}_{2}(\mathrm{EA}=1.023 \pm 0.007 \mathrm{eV}$ [12]) is smaller than the dissociation energy of $\mathrm{Ag}_{2}^{-}\left(D_{0}=\right.$ $1.37 \pm 0.16 \mathrm{eV}[12])$, there are rovibrational levels for which electron emission is the only possible decay path, and the decay from such levels is therefore the only possible explanation for the presently observed electron emission. In the left panel of Fig. 3, a single vibrational level $(v=110)$ of the electronic ground state with $L=0$ is drawn as an example. For that particular level, energetically possible electron emission implies that the resulting neutral dimer has a vibrational quantum number of $v \leq 10$. This illustrates that the energy transferred to the electron is initially stored as vibrational excitation in the dimer anion and that the emission of the electron is accompanied by a sudden, dramatic decrease of the vibrational excitation. The total kinetic energy of the two nuclei in the center-of-mass frame instantaneously decreases by about $1 \mathrm{eV}$ for this particular 
case, as can be seen in the left panel of Fig. 3. Note that for the process to be energetically allowed the internuclear distance is restricted to a range where the summed kinetic energy of the two nuclei (in their center-of-mass system) is high. As no diabatic potential-energy curve crossings are available to drive the energy transfer from the nuclear to the electronic degrees of freedom, the electron-emission process that we observe is a dramatic example of a departure from a BornOppenheimer description of the dynamics.

As mentioned above, the ions decaying by fragmentation have, in part, different rovibrational excitations than those decaying by electron emission. In order to investigate this in more detail, we calculate the rovibrational energy level structure of neutral and anion silver dimers. We again use the $\mathrm{Ag}_{2}$ and $\mathrm{Ag}_{2}^{-}$Morse potentials based on parameters from Refs. [12,22], and for each value of angular momentum $L$, we solve the radial Schrödinger equation numerically to find the vibrational levels as the eigenstates for that given potential, including the centrifugal distortion. The energy of each rovibrational level is then compared to both the energy of the separated $\mathrm{Ag}^{-}$ion and $\mathrm{Ag}$ atom and the lowest vibrational level of the neutral dimer with the same value of $L$ as the dimer anion. The emitted electron can only carry away a few quanta of angular momentum and here the angular momentum of the electron is ignored. This way we determine the possible decay paths for each rovibrational level.

In Fig. 4, we show a map over the rovibrational levels of $\mathrm{Ag}_{2}^{-}$, indicating whether they are stable or if they can decay by fragmentation, electron emission, or both. The dimer anions are formed in the ion source in violent collisions of $6 \mathrm{keV} \mathrm{Cs}^{+}$ions on a silver surface and have been found to occupy a wide range of rovibrational levels [23]. Indeed, the presently observed decays and the large component of stable (or extremely long-lived) $\mathrm{Ag}_{2}^{-}$anions suggest a very broad rovibrational distribution.

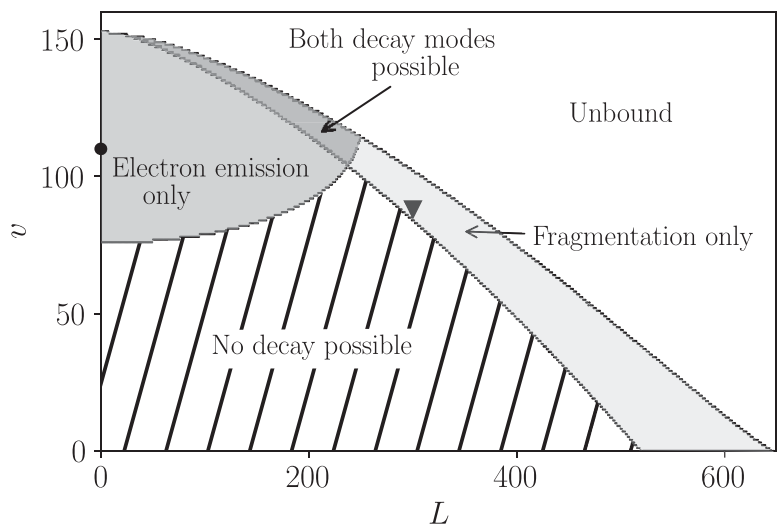

FIG. 4. A map of the rovibrational levels of $\mathrm{Ag}_{2}^{-}$indicating for which combinations of rotational and vibrational excitations fragmentation and/or electron emission are energetically allowed. The dot and triangle markings indicate the $L$ and $v$ corresponding to the levels drawn in the left and right panels of Fig. 3, respectively.
We argued above that the dramatic decrease in vibrational excitation accompanying the electron emission is particularly remarkable as no avoided crossing of the two involved adiabatic potential-energy curves is available to drive the transition. In a quantum mechanical description of electron emission, the nonadiabatic coupling between the anion and the ionization continuum will give rise to coupling elements of the form [13] $-\hbar^{2} /(2 \mu)\left\langle\chi_{v^{\prime} L^{\prime}}^{i}\left|f_{i j}(R) d / d R\right| \chi_{v L}^{j}\right\rangle$, where $\mu$ is the reduced mass of the nuclei and $\chi_{v^{\prime} L^{\prime}}^{i}$ and $\chi_{v L}^{j}$ are the nuclear wave functions of a given rovibrational state of $\mathrm{Ag}_{2}$ and $\mathrm{Ag}_{2}^{-}$, respectively. The nonadiabatic coupling element $f_{i j}(R)$ is strictly zero in the Born-Oppenheimer approximation, but here we formally express it as $f_{i j}(R)=$ $\left\langle\psi_{i}|\partial / \partial R| \psi_{j}\right\rangle$, where $\psi_{i}$ and $\psi_{j}$ are the full electronic wave functions of the neutral plus a free electron and of the anion, respectively. The nonadiabatic coupling is, in general, large when the adiabatic potential-energy curves are close in energy - i.e., at avoided crossings. Here the potential curves of the $\mathrm{Ag}_{2}^{-}$and $\mathrm{Ag}_{2}$ ions are far apart in energy for all values of $R$ and, as a result, $f_{i j}$ will be small (but not zero) and not a strong function of $R$.

In spite of this weak coupling, the electron emission process dominates the measured decay signal for $t>100 \mathrm{~ms}$. In fact, electron emission events account for $13 \%$ of the total number of decay events during the $10 \mathrm{~s}$ observation time. This surprising observation relies on the exceptional experimental conditions and the absence of any other decay channels rather than on the efficiency of the electron emission process. To quantify the latter, we use the detailed balance approach $[24,25]$ to estimate the cross section for the time-reversed process - the attachment of a free electron to the neutral dimer via a strong increase of the vibrational excitation of the dimer system. Assuming an electron energy of $20 \mathrm{meV}$ (a typical vibrational spacing) and a decay rate of $10 \mathrm{~s}^{-1}$, which is characteristic of the measured timescale, indicates an extremely small electron attachment cross section of the order of $10^{-9} \AA^{2}$. This semiempirical cross section estimate is about 9 orders of magnitude smaller than the geometrical cross section of the dimer, which illustrates the weakness of the coupling.

In conclusion, we have investigated the decay of internally hot silver dimer anions stored in a cryogenic electrostatic ion-beam-storage ring with exceptionally low residual-gas density. By separately detecting neutral and charged products of the decay, we demonstrate experimentally that fragmentation is the dominating decay process for the initial parts of the decay (up to $100 \mathrm{~ms}$ of storage). Further, we unambiguously identify a prominent contribution from electron emission. This is remarkable as no potential-energy curve crossings are available to mediate a coupling between the nuclear and electronic degrees of freedom. Thus, the presently observed electron emission from the homonuclear dimer anion $\mathrm{Ag}_{2}^{-}$represents a 
dramatic deviation from a Born-Oppenheimer description of the decay.

This work was performed at the Swedish National Infrastructure, DESIREE (Swedish Research Council Contract No. 2017-00621). Furthermore, H. C., H. Z., and H.S. thank the Swedish Research Council for individual project Grants No. 2019-04379, No. 2016-04181, and No. 2018-04092. A. S. acknowledges support by the Austrian Science Fund FWF within the DK-ALM (W1259-N27).

*emma.anderson@fysik.su.se †henning.schmidt@fysik.su.se

[1] M. Born and R. Oppenheimer, Zur quantentheorie der molekeln, Ann. Phys. (N.Y.) 389, 457 (1927); for an informal English translation, see https://www2.ulb.ac.be/ cpm/people/bsutclif/bornopn_corr.pdf.

[2] S. B. Nielsen, A. Lapierre, J. U. Andersen, U. V. Pedersen, S. Tomita, and L. H. Andersen, Absorption Spectrum of the Green Fluorescent Protein Chromophore Anion In Vacuo, Phys. Rev. Lett. 87, 228102 (2001).

[3] N. Kono, T. Furukawa, H. Tanuma, J. Matsumoto, H. Shiromaru, T. Azuma, K. Najafian, M. S. Pettersson, B. Dynefors, and K. Hansen, Inverse internal conversion in $\mathrm{C}_{4}{ }^{-}$below the electron detachment threshold, Phys. Chem. Chem. Phys. 17, 24732 (2015).

[4] Y. Ebara, T. Furukawa, J. Matsumoto, H. Tanuma, T. Azuma, H. Shiromaru, and K. Hansen, Detection of Recurrent Fluorescence Photons, Phys. Rev. Lett. 117, 133004 (2016).

[5] N. Kono, R. Suzuki, T. Furukawa, J. Matsumoto, H. Tanuma, H. Shiromaru, T. Azuma, and K. Hansen, Electronic and vibrational radiative cooling of the small carbon clusters $\mathrm{C}_{4}{ }^{-}$and $\mathrm{C}_{6}{ }^{-}$, Phys. Rev. A 98, 063434 (2018).

[6] S. Martin, J. Bernard, R. Brédy, B. Concina, C. Joblin, M. Ji, C. Ortega, and L. Chen, Fast Radiative Cooling of Anthracene Observed in a Compact Electrostatic Storage Ring, Phys. Rev. Lett. 110, 063003 (2013).

[7] E. K. Anderson, M. Kamińska, K. C. Chartkunchand, G. Eklund, M. Gatchell, K. Hansen, H. Zettergren, H. Cederquist, and H. T. Schmidt, Decays of excited silvercluster anions $\operatorname{Ag}_{n}, n=4$ to 7 , in the double electrostatic ion ring experiment, Phys. Rev. A 98, 022705 (2018).

[8] K. Hansen, M. H. Stockett, M. Kaminska, R. F. Nascimento, E. K. Anderson, M. Gatchell, K. C. Chartkunchand, G. Eklund, H. Zettergren, H. T. Schmidt, and H. Cederquist, Spontaneous decay of small copper-cluster anions $\mathrm{Cu}_{n}{ }^{-}(n=3-6)$, on long time scales, Phys. Rev. A 95, 022511 (2017).

[9] B. Kafle, O. Aviv, V. Chandrasekaran, O. Heber, M. L. Rappaport, H. Rubinstein, D. Schwalm, D. Strasser, and D. Zajfman, Electron detachment and fragmentation of laserexcited rotationally hot $\mathrm{Al}_{4}{ }^{-}$, Phys. Rev. A 92, 052503 (2015).
[10] S. Menk, S. Das, K. Blaum, M. W. Froese, M. Lange, M. Mukherjee, R. Repnow, D. Schwalm, R. von Hahn, and A. Wolf, Vibrational autodetachment of sulfur hexafluoride anions at its long-lifetime limit, Phys. Rev. A 89, 022502 (2014).

[11] E. J. Heller, The many faces of tunneling, J. Phys. Chem. A 103, 10433 (1999).

[12] J. Ho, K. M. Ervin, and W. C. Lineberger, Photoelectron spectroscopy of metal cluster anions: $\mathrm{Cu}_{n}^{-}, \mathrm{Ag}_{n}^{-}$, and $\mathrm{Au}^{-}{ }_{n}$, J. Chem. Phys. 93, 6987 (1990).

[13] N. Douguet, S. F. dos Santos, M. Raoult, O. Dulieu, A. E. Orel, and V. Kokoouline, Theoretical study of radiative electron attachment to $\mathrm{CN}, \mathrm{C}_{2} \mathrm{H}$, and $\mathrm{C}_{4} \mathrm{H}$ radicals, J. Chem. Phys. 142, 234309 (2015).

[14] C. Chin, R. Grimm, P. Julienne, and E. Tiesinga, Feshbach resonances in ultracold gases, Rev. Mod. Phys. 82, 1225 (2010).

[15] H. T. Schmidt et al., First storage of ion beams in the Double Electrostatic Ion-Ring Experiment: DESIREE, Rev. Sci. Instrum. 84, 055115 (2013).

[16] R. D. Thomas et al., The double electrostatic ion ring experiment: A unique cryogenic electrostatic storage ring for merged ion-beams studies, Rev. Sci. Instrum. 82, 065112 (2011).

[17] E. Bäckström, D. Hanstorp, O. M. Hole, M. Kaminska, R. F. Nascimento, M. Blom, M. Björkhage, A. Källberg, P. Löfgren, P. Reinhed, S. Rosén, A. Simonsson, R. D. Thomas, S. Mannervik, H. T. Schmidt, and H. Cederquist, Storing keV Negative Ions for an Hour: The Lifetime of the Metastable ${ }^{2} P_{1 / 2}^{o}$ level in ${ }^{32} S^{-}$, Phys. Rev. Lett. 114, 143003 (2015).

[18] J. M. Peek, A. Hashemi Attar, and C. L. Beckel, Spontaneous emission lifetimes in the ground electronic states of $\mathrm{HD}^{+}$and $\mathrm{H}_{2}{ }^{+}$, J. Chem. Phys. 71, 5382 (1979).

[19] S. P. Møller, ELISA, an electrostatic storage ring for atomic physics, Nucl. Instrum. Methods Phys. Res., Sect. A 394, 281 (1997).

[20] J. Fedor, K. Hansen, J. U. Andersen, and P. Hvelplund, Nonthermal Power Law Decay of Metal Dimer Anions, Phys. Rev. Lett. 94, 113201 (2005).

[21] K. Hansen, J. U. Andersen, P. Hvelplund, S. P. Møller, U. V. Pedersen, and V. V. Petrunin, Observation of a $1 / t$ Decay Law for Hot Clusters and Molecules in a Storage Ring, Phys. Rev. Lett. 87, 123401 (2001).

[22] M. D. Morse, Clusters of transition-metal atoms, Chem. Rev. 86, 1049 (1986).

[23] A. Wucher and B. J. Garrison, Cluster formation in sputtering: A molecular dynamics study using the MD/MCcorrected effective medium potential, J. Chem. Phys. 105, 5999 (1996).

[24] V. Weisskopf, Statistics and nuclear reactions, Phys. Rev. 52, 295 (1937).

[25] K. Hansen, Statistical Physics of Nanoparticles in the Gas Phase, Springer Series on Atomic, Optical, and Plasma Physics Vol. 73 (Springer, Dordrecht, 2013). 